\title{
Regional Cerebral Blood Flow in Children From 3 to 5 Months of Age
}

\author{
A.F. Duncan, A. Caprihan, E.Q. Montague, J. Lowe, R. Schrader, and J.P. Phillips
}

\begin{abstract}
BACKGROUND AND PURPOSE: Understanding the relationship between brain and behavior in early childhood requires a probe of functional brain development. We report the first large study of regional CBF by use of arterial spin-labeling in young children.
\end{abstract}

MATERIALS AND METHODS: Cerebral blood flow by use of arterial spin-labeling was measured in 61 healthy children between the ages of 3 and 5 months. Blood flow maps were parcellated into 8 broadly defined anatomic regions of each cerebral hemisphere.

RESULTS: There was no sex effect; however, group analysis demonstrated significantly greater CBF in the sensorimotor and occipital regions compared with dorsolateral prefrontal, subgenual, and orbitofrontal areas $(P<.0001)$. A significant age effect was also identified, with the largest increase in blood flow between 3 and 5 months occurring in the following regions: orbitofrontal $(P<.009)$, subgenual $(P<$ $.002)$, and inferior occipital lobe $(P=.001)$.

CONCLUSIONS: These results are consistent with prior histologic studies demonstrating regional variation in brain maturation and suggest that arterial spin-labeling is sensitive to regional as well as age-related differences in CBF in young children.

ABBREVIATION: ASL $=$ arterial spin-labeling

C

hild development during the first year of life is dynamic and complex, with well-established clinical norms for monitoring behavioral development. ${ }^{1}$ Brain structural development at this age is characterized by changing synaptic attenuation, ${ }^{2}$ neuronal attenuation, ${ }^{3}$ and myelination. ${ }^{4}$ Less is known about functional brain development, which prevents a full understanding of the relationships between brain structure and the increasing cognitive and motor skills that characterize early childhood.

Brain function can be assessed with perfusion studies because

\footnotetext{
Received April 4, 2013; accepted after revision June 8.

From the Department of Pediatrics, Division of Neonatology (A.F.D., J.L.), Clinical and Translational Science Center (R.S.), and Department of Neurology (J.P.P.), University of New Mexico, Albuquerque, New Mexico; The MIND Research Network (A.C.), Albuquerque, New Mexico; and NeuroDevelopmental Science Center (E.Q.M.), Akron Children's Hospital, Akron, Ohio.

Primary funding was provided by Dr George Cowan and the Delle Foundation. This project was supported in part or in full by the National Center for Research Resources and the National Center for Advancing Translational Sciences of the $\mathrm{Na}$ tional Institutes of Health through Grant No. 8ULITR000041 and the University of New Mexico Clinical and Translational Science Center Translational Sciences of the National Institutes of Health through Grant No. 8UL1TR000041. Additional support included a grant from the Department of Energy DE-FG02-08ER64581.

Please address correspondence to Andrea Freeman Duncan, MD, The University of New Mexico, Department of Pediatrics, MSC10 5590, One University of New Mexico, Albuquerque, NM 87131-0001; e-mail: afduncan@salud.unm.edu

- Indicates open access to non-subscribers at www.ajnr.org

http://dx.doi.org/10.3174/ajnr.A3728
}

of the tight coupling of metabolism to blood flow. Regional metabolism can be directly measured by use of PET, which requires radioactively labeled water as an injected tracer. In children 3 months of age, PET demonstrates increased metabolism in the parietal, temporal and visual cortex, basal ganglia, and cerebellar hemispheres, with increased metabolism in the frontal lobes later in the first year. ${ }^{5}$ The radiation required for PET limits its feasibility for use in studies of typically developing children. ${ }^{6}$

Recent advances in MR technology provide a noninvasive method of assessing regional CBF by use of arterial spin-labeling (ASL). ${ }^{7}$ Because there is no radiation or injectable contrast agent, ASL is appropriate for longitudinal studies in children. Preliminary work suggests that it is sensitive to changes occurring during normal brain maturation ${ }^{7,8}$ as well as in clinical populations such as children with preclinical sickle cell disease. ${ }^{9}$ However, normal regional CBF has not been established by use of ASL. Although there is tremendous potential for ASL to provide early markers of disease or perhaps even therapeutic targets in young children with various clinical conditions, a necessary first step is identifying the range of normal CBF in healthy children.

The objectives of this study were to quantify normal regional CBF through the use of ASL in healthy term-born children 3-5 months of age and to determine whether age-related changes in $\mathrm{CBF}$ could be detected. At this age, tremendous developmental changes are occurring, which may be reflected in regional brain metabolism. 


\section{MATERIALS AND METHODS \\ Subjects}

This cross-sectional study was part of an ongoing longitudinal investigation of normal child development; 77 subjects were studied at $4 \pm 1$ month of age. Imaging occurred at the MIND Research Network in Albuquerque, New Mexico. Children were recruited from pediatric clinics and community advertisements. The study was approved by the University of New Mexico Institutional Review Board, and informed consent obtained from parents of all children before participation. Inclusion criteria included uncomplicated term delivery and no known medical or developmental disorders. Exclusion criteria included genetic disorders, visual or hearing impairment, or contraindication to MR imaging.

\section{Data Acquisition}

MRI was performed at night during natural sleep without sedative medication. Caregivers remained with children throughout. Once asleep, headphones were placed over the infants' ears for noise protection. Imaging was performed on a Trio 3T MR imaging scanner (Siemens, Erlangen, Germany) by use of the provided 12-channel radio frequency head coil.

T1-weighted images were collected along the sagittal plane with the use of a 5-echo 3D MPRAGE sequence: $\mathrm{TR}=2530$; $\mathrm{TE}=$ $1.64,3.5,5.36,7.22,9.08 ; \mathrm{TI}=1200$, flip angle $=7^{\circ} ; \mathrm{FOV}=256 \times$ $256 \mathrm{~mm}$; matrix $=256 \times 256,1$-mm-thick section, 192 sections; with generalized auto-calibrating partially parallel acquisition acceleration factor 2 .

CBF was measured by means of the pulsed ASL method. ${ }^{10,11}$ Pulse sequence parameters were FOV $=220 \mathrm{~mm}, 64 \times 64$ matrix, and 4-mm section thickness with a 1-mm gap. Twenty-four axial sections were taken in ascending order along the anteroposterior commissure; $\mathrm{TR}=4000 \mathrm{~ms}$ and $\mathrm{TE}=12 \mathrm{~ms}$. The sequence consisted of 105 volumes of interleaved global and section-selective inversion recovery acquisition with a saturation pulse applied to a slab inferior and superior to the imaging sections. The delay between the inversion pulse and saturation pulses were TI1 $=600$ $\mathrm{ms}$, and the delay between the inversion and excitation pulses was TI2 $=1600 \mathrm{~ms}$. Total acquisition time was 7 minutes.

\section{Data Processing}

Because tissue contrast by use of $\mathrm{T} 1$ alone in children 4 months of age may be limited, we did not use an automatic segmentation algorithm such as FreeSurfer (http://surfer.nmr.mgh.harvard. edu). ${ }^{11}$ We instead optimized the T1 parameters to give improved contrast in the acquired images and used a 9-month template. ${ }^{12}$ The 9-month template was first separated into right and left hemispheres. Each hemisphere was parcellated into 8 regions by use of methods described by Limperopoulos et al. ${ }^{13}$ The axial plane was segmented through the anterior and posterior commissures. Three coronal planes were then manually drawn to divide each hemisphere into the following anatomic regions: dorsolateral prefrontal, premotor, sensorimotor, parieto-occipital, orbito-frontal, subgenual, midtemporal, and inferior-occipital (Fig $1 B$ ). The choice of the 3 coronal planes follows the locations used by Limperopoulos et al. ${ }^{13}$ The regions shown on one sagittal section in Fig $1 B$ are volumes comprising the entirety of the cerebral volume. These regions were defined once on the 9-month tem-
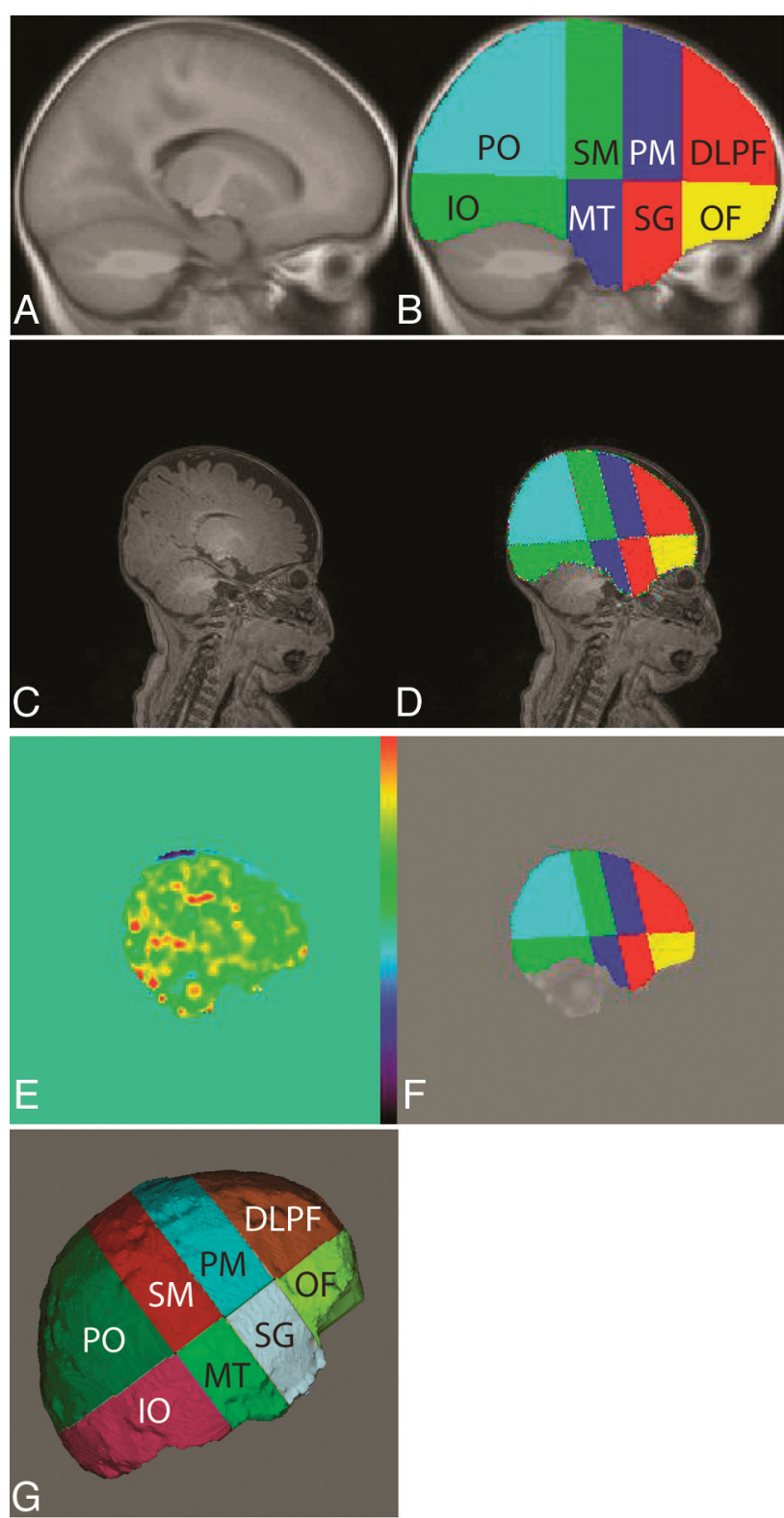

FIG 1. $A$ and $B$, Brain segmentation process of the 9-month template is shown; $C$, 4-month $T 1$ image; $D$, corresponding atlas after mathematica transformation. $E, C B F$ image; $F, C B F$ image with the atlas overlaid to demonstrate how well they are registered; $G$, the 8 segments projected onto the cortical surface.

plate and were then transformed on the T1 image obtained on each subject. Figure $1 C$ shows a T1 image of the 4-month-old infant brain. The 4-month image was spatially normalized to the 9-month template by trimming the 4-month brain below the neck and extracting it by use of the Brain Extraction Tool program in the Functional MR Imaging of the Brain Software Library (http:// fsl.fmrib.ox.ac.uk/fsl) package. The image was then spatially normalized by use of the FMRIB Linear Image Registration Tool program.

The 16 regions marked on the template ( 8 matching regions in each hemisphere) were transformed to the 4 -month image by use of the inverse of the transformation found in the previous step. The resulting 8 regions of the right hemisphere mapped onto the 4-month brain are shown in Fig $1 D$. This method was repeated for 
Demographic information for study subjects

\begin{tabular}{lccc}
\hline & Total $(\boldsymbol{n}=\mathbf{6 1})$ & Male $(\boldsymbol{n}=\mathbf{3 7}) \mathbf{( 6 1 \% )}$ & Female $(\boldsymbol{n}=\mathbf{2 4})(\mathbf{3 9 \%})$ \\
\hline Birth weight, g & $3,294.95 \pm 432.2$ & $3,363.6 \pm 408.8$ & $3,190.5 \pm 554.6$ \\
Gestational age, wk & $39.2 \pm 1.4$ & $37.3 \pm 9.1$ & $38.9 \pm 1.5$ \\
Age at scanning, d & $123.6 \pm 13$ & $122.3 \pm 12.6$ & $125.5 \pm 13.4^{\mathrm{a}}$ \\
Sex, \% girls & $39 \%$ & & \\
Ethnicity $^{\mathrm{a}}$ & & & \\
White & $15(24.59 \%)$ & $8(21.62 \%)$ & $7(29.16 \%)$ \\
Black & $1(1.64 \%)$ & $1(2.70 \%)$ & $0(0 \%)$ \\
Hispanic & $34(55.73 \%)$ & $21(56.75 \%)$ & $13(54.16 \%)$ \\
Native American & $3(4.92 \%)$ & $2(5.40 \%)$ & $1(4.16 \%)$ \\
Other & $4(6.55 \%)$ & $3(8.10 \%)$ & $1(4.16 \%)$ \\
Unknown & $4(6.55 \%)$ & $2(5.40 \%)$ & $2(8.33 \%)$ \\
\hline
\end{tabular}

Note:-Values are depicted as means \pm standard deviations unless otherwise indicated. ${ }^{\text {a }} P<.05$.

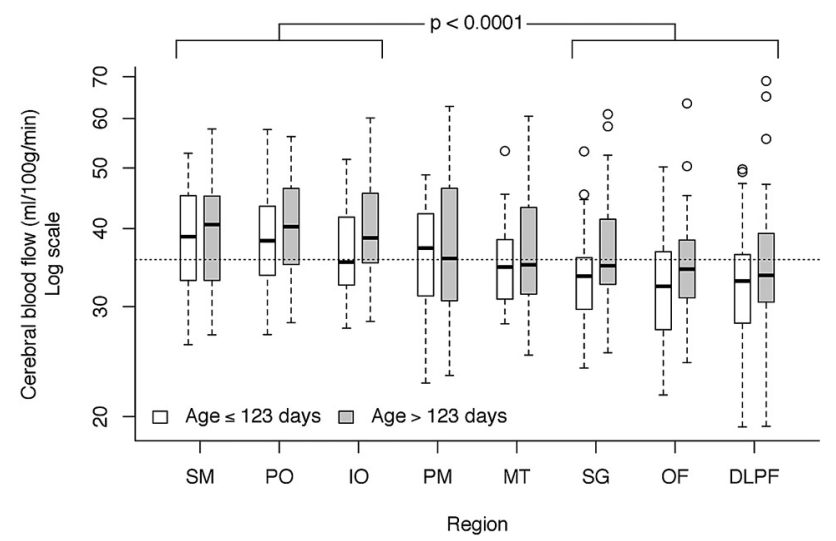

FIG 2. Regional differences in CBF. Although there were no significant differences within the 3 higher flow regions (sensorimotor, parietooccipital, and inferior-occipital; $P=.43$ ) or within the 3 lower flow regions (subgenual, orbito-frontal, and dorsolateral prefrontal; $P=$ .15), mean CBF between the high and low groups was highly significant. The mixed model fit age in a continuous manner, but, for illustrative purposes, age is split at the median. Horizontal dotted line is the median CBF value across all regions and ages.

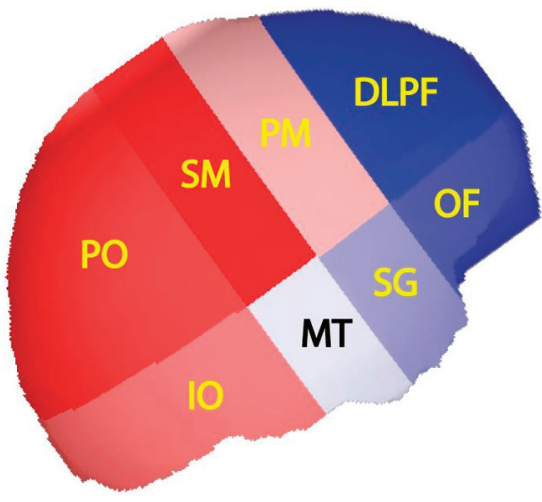

FIG 3. Regions with CBF above study population median are shown in shades of red and those regions with CBF below the median are shown in shades of blue. Darker color indicates greater deviation from the median flow. Thus, the sensorimotor (SM) region had the highest flow above the median and the dorsolateral prefrontal (DLPF) region had the lowest flow below the median.

all subjects. We proportionally divided each subject's brain into the 16 regions. Regions were labeled to identify the location in the brain. Thus, the dorsolateral prefrontal cortex is the superiorfrontal region of the brain of identical proportional volume for each child.
The raw ASL images were motion-corrected by registering all the images to the first ASL image. The CBF image was created by use of methods described by Wang et al. ${ }^{10}$ Mean CBF was calculated for the each of the 16 regions defined on the T1 image (Fig $1 D)$. Figure $1 E$ demonstrates the CBF image spatially normalized to the T1 image, with overlaid regions of interest shown in Fig $1 F$. Figure $1 G$ shows a cortical projection of the regions.

\section{Statistical Analyses}

Data for the repeated measures of mean total CBF on 8 regions (dorsolateral prefrontal, inferior-occipital, midtemporal, orbitofrontal, premotor, parieto-occipital, subgenual, sensorimotor) were plotted through the use of profile and boxplots to identify outliers. One subject was excluded because of extremely low mean CBF values across all regions. There were other isolated high outliers; none were removed, but data were analyzed on logarithmic scale to control their effect. No difference was found between mean $\mathrm{CBF}$ of left and right hemispheres for any region; therefore, right and left hemispheres were combined for analysis. Repeated measures were fit with linear mixed models in Proc Mixed in Statistical Analysis Software version 9.3 (SAS Institute, Cary, North Carolina) by use of a completely symmetric covariance structure within subjects. Fixed effects fit were region, age, and sex, with all possible 2-way interactions between them. Tukey-Kramer adjustments for multiple comparisons were used to identify groupings among regions. Estimated effects were exponentiated to return to original units.

\section{RESULTS \\ Participants}

Of 77 children scanned, 61 provided acceptable ASL data, for a success rate of $74 \%$. There were significantly more boys than girls ( $n=37$ versus $24, P<.001)$. Ethnicities included Hispanic (56\%), white (25\%), Native American (5\%), black (2\%), and other (7\%). Four children were of unknown ethnicity. Age at time of scanning ranged from 90-150 days (Table 1).

Figure 2 illustrates the regional distribution of CBF among the 8 regions. There were no significant 2 -way interactions between sex, region, and age. Sex was not significant $(P=.70)$, though region $(P<.0001)$ and age $(P=.014)$ were significant. Tukey comparisons found no significant differences within the 3 higher flow regions (sensorimotor, parieto-occipital, and inferior-occipital; $P=.43$ ) or within the 3 lower flow regions (subgenual, orbito-frontal, and dorsolateral prefrontal; $P=.15$ ). However, when comparing $\mathrm{CBF}$ between the higher and lower flow regions, all differences were significant. A contrast test for differences between average CBF in the high and low group was highly significant $(P<.0001)$. There was no clear placement of premotor and midtemporal regions into one of these groups or into a separate group. Figure 3 shows the relative anatomic location and CBF of the regions. The median subject age was 123 days. At this age, the (geometric) mean for the high-flow regions was $38.9 \mathrm{~mL} / 100$ $\mathrm{g} / \mathrm{min}(95 \% \mathrm{CI}, 37.1,40.8)$, and $34.2 \mathrm{~mL} / 100 \mathrm{~g} / \mathrm{min}(32.6,35.9)$ 


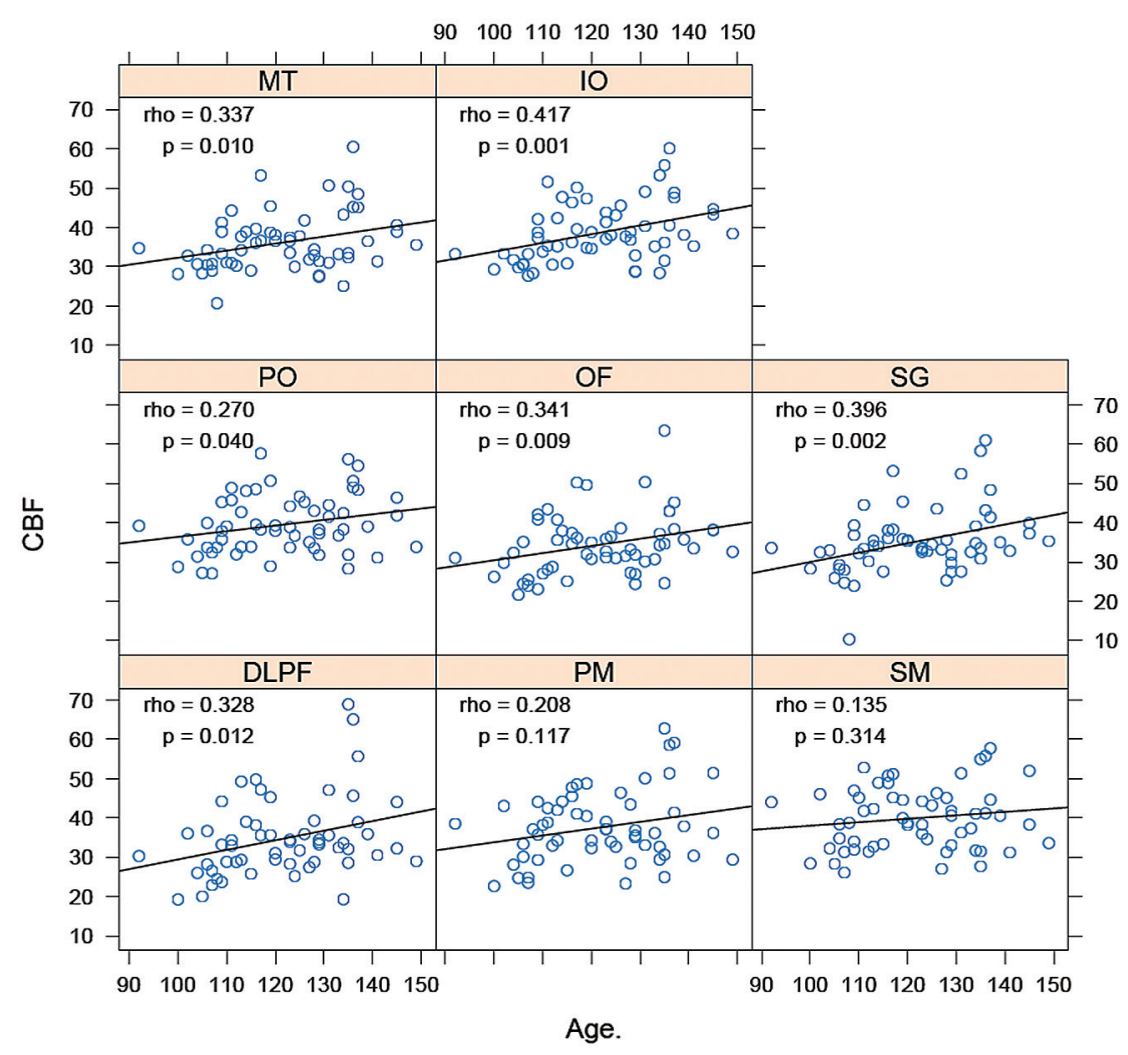

FIG 4. Cerebral blood flow versus age within each region; $\rho$ is Spearman rank correlation coefficient.

for the low-flow regions. This corresponds to an average $13.8 \%$ $(11.1 \%, 15.5 \%)$ difference between high- and low-flow regions.

An age effect was also noted. Average CBF increased $14.9 \%$ for each 30-day age increase; however, there was regional variation ranging from 3.0-28.1\% (Fig 4). The relationship between age and CBF change was significant in all areas except premotor and sensorimotor. The strongest correlation between age and CBF was seen in the following areas: orbito-frontal $(P=.009)$, inferioroccipital $(P=.001)$, and subgenual $(P=.002)$.

\section{DISCUSSION}

Understanding the relationship between brain and behavior in early childhood requires a probe of functional brain development. Because CBF reflects regional metabolism, ASL offers a noninvasive approach to studying brain function. In this report, we demonstrate the feasibility of the use of ASL in a group of healthy infants. Even within the narrow age range studied, ASL identifies significantly greater CBF in occipital and parietal lobes and the primary sensorimotor cortex compared with more anterior brain regions. In addition, we report significant age-related changes in $\mathrm{CBF}$, which suggests that CBF change over time may be an important measure of functional brain development.

Histologic studies demonstrate the dynamic nature of early brain development, with rapid changes in neuronal ${ }^{3}$ and synap$\mathrm{tic}^{2}$ attenuation occurring first in primary sensory (primary visual cortex, Heschl gyrus) and later in higher-order association regions (prefrontal). Recent MR imaging studies by use of highresolution sequences show a similar pattern in early adolescence ${ }^{14}$ and as early as the first 2 months of life. ${ }^{15}$ More recent techniques, such as DTI, characterize structural white matter development through childhood. ${ }^{16}$ These histologic and imaging studies provide powerful insights into the complex nature of early structural brain development.

Structure, however, does not equal function. Although our understanding of the rich tapestry of structural brain development has increased, the relation to brain function and behavior remains unclear. A study of functional brain development by use of PET to assess regional cerebral glucose metabolism ${ }^{5,17}$ found that the primary sensory and motor cortices, along with deeper cerebral areas, had the highest metabolic rates during the first months of life. Next, the parietal and temporal cortex developed increased glucose utilization. The frontal cortex demonstrated increased glucose metabolism by the end of the first year. These studies were ethically possible because of the sole inclusion of children requiring PET for clinical reasons that involve the substantial radiation exposure. $\mathrm{PET}$ is not feasible for the study of normal brain development. Other functional imaging modalities require radiation (SPECT) or subject cooperation (stimulusevoked fMRI). Recently, functional connectivity MR imaging has been used to identify network connectivity in children by assessment of the coherence of fluctuating levels of blood oxygenation at rest. ${ }^{18}$

Tight coupling of brain metabolism and blood flow ${ }^{19}$ suggests that CBF can be used as a marker of regional metabolic activity. With the use of magnetically labeled arterial water as a tracer, ASL quantifies CBF noninvasively. ${ }^{20}$ ASL has been used for study of brain changes in disease states including vascular disease, dementia, and epilepsy. ${ }^{20}$ Noguchi et $\mathrm{al}^{21}$ demonstrated that increased CBF measured by ASL may be related to increased microvascular attenuation in subjects with brain tumors. It is possible that in healthy children, increased CBF may be related to increased regional synaptogenesis, which, through neurovascular coupling, increases regional microvascular attenuation. Because ASL is noninvasive and requires no external tasks or exposure to radiation, it is well suited for use in pediatric samples. Wang et $\mathrm{al}^{10}{ }^{10}$ were the first to use ASL in children, establishing feasibility at 1.5T. More recently, ASL has shown that preterm children have higher perfusion than term children. ${ }^{22}$ In healthy individuals from 4 years to adulthood, there is a decrease in $\mathrm{CBF}$ - most prominent in gray matter-by midadolescence. ${ }^{8}$ Taki and Kawashima ${ }^{23}$ reported a nonlinear relationship between age and CBF from 5-18 years. They noted that these findings were similar to the reported gray matter volume increase in prepuberty followed by a postpuberty decrease. ${ }^{24}$ Wang et al ${ }^{10}$ compared 8 children at 7 and 13 months of age. Regional CBF increased significantly at 13 
months in the dorsolateral frontal lobes and decreased in the sensorimotor areas, consistent with the PET studies of Chugani. ${ }^{5,17}$

We report the first large study of regional CBF by using ASL in young children. Lack of MR imaging contrast between gray and white matter at this age prohibited detailed regional segmentation, however by dividing the brain into broad anatomic areas ${ }^{13}$ we were able to demonstrate significantly greater CBF in the sensorimotor cortex and parietal/occipital lobes compared with dorsolateral prefrontal, orbitofrontal, and subgenual regions. Children were 3-5 months of age and therefore it is difficult to compare our findings with those of Limperopoulos et $\mathrm{al}^{13}$ However, our findings are consistent with the prior PET work of Chugani, ${ }^{5}$ who found increased cerebral metabolism at this age in the parietal and occipital regions and reduced frontal lobe metabolism. Our findings are also consistent with histologic studies demonstrating primary sensorimotor regions maturing earlier than higher association regions. ${ }^{2,3}$

Our cohort included 61 subjects 3-5 months of age, allowing us to evaluate regional change in $\mathrm{CBF}$ over time. We found that CBF increased significantly with age in every region except the premotor and sensorimotor regions. Two of the regions in which there was the greatest age effect (orbital frontal and subgenual) had lower overall CBF. We speculate that this changing blood flow may reflect increasing metabolism that precedes a corresponding maturation of brain structure as occurs in the more anterior brain regions toward the end of the first year of life. Further studies are required to determine whether regional changes in CBF predict structural development, which could potentially serve as a marker of abnormal development before it becomes clinically evident.

Our findings are also consistent with normal clinical development. Clinical skills developing at 3-5 months include visualmotor tasks such as reaching, rolling over, and increasing control of movement. ${ }^{25}$ Facial recognition and social interaction are highly visually dependent and are actively developing at this stage of childhood. ${ }^{26}$ These skills involve the sensorimotor and occipital/parietal regions, which had the highest CBF in our subjects. The lowest flow was found in the anterior brain regions, which subserve later developing skills such as working memory, impulse inhibition, and self-regulation. ${ }^{27,28}$

We did not find a sex difference in CBF at this age; however only $39 \%$ of our study cohort were girls. This is a limitation that may have been partially mitigated by our relatively large sample size, but the role of sex in early CBF requires further evaluation. Likewise, our cohort reflected the local demographics with a proportionally large Hispanic population. Although this may be a limitation, we are aware of no evidence that ethnicity affects regional CBF. Our unsuccessful scans were largely caused by excessive movement; thus, children who had difficulty sleeping in our MR imaging environment were not included in the data analysis. This is a difficult limitation to avoid. By use of an experienced scanning team and providing families with multiple options for aiding sleep, our success rate of $74 \%$ probably reflects a range of normally developing children. Though our sample size was substantial, individual variability in brain development at this age probably warrants a larger sample size to ensure power to detect all differences of clinical import. In addition, data obtained from the cerebellum was markedly inconsistent, and therefore this region was not included in our study. Finally, this study is limited by the broadly defined regions studied, the inclusion of gray and white matter in the measurements, and the use of only $\mathrm{T} 1 \mathrm{se}-$ quences. With additional data from a T2 sequence, a more physiologically meaningful segmentation may have been possible. These limitations must be considered in the interpretation of our results; however, the consistency of the findings across the study group strengthens the possibility that these differences may not have contributed importantly to the findings. However, it is impossible to know how these factors may bias our results. The validity of our findings is strengthened by their consistency with those found in a previous study of regional cerebral glucose utilization by use of PET scanning. ${ }^{5,17}$ This implies that ASL may provide a noninvasive means of determining regional brain functioning with the same accuracy as PET scanning without radiation exposure.

Our findings highlight the potential for use of ASL to assess functional brain development in early childhood. Future longitudinal studies including motor and cognitive measures may offer insights into early brain/behavioral relationships that have relevance for understanding normal and abnormal child development. Although these studies are inherently difficult, given the challenges of scanning children at this age without sedation, ASL offers the possibility of establishing early markers of abnormal development that may allow earlier, more effective treatment for children at risk for developmental disorders.

\section{CONCLUSIONS}

This study provides valuable information regarding neuroanatomic development in infants. The data begin to fill a large gap in the literature in the area of normal cerebral development. Knowledge of the normal development of the human brain is vital because without normative measures, it is impossible to truly characterize abnormalities of cerebral development. A better understanding of brain development will afford the medical community the opportunity to leverage the innate neuroplasticity involved in early brain development ${ }^{29}$ by providing targeted developmental interventions at earlier ages to improve outcomes.

\section{ACKNOWLEDGMENTS}

We would like to acknowledge the work of our doctoral students and MIND Research Network staff: Susanne Duvall, Joy Van Meter, Lynette Silva, Judith Segall, Diana South, and Cathy Smith, who worked many nights performing MR imaging scanning on the children. We would also like to thank the families who traveled long distances to the University of New Mexico and persevered late into the night to get their children to sleep through the scans used to complete this study.

Disclosures: Arvind Caprihan—RELATED: Grant: Delle Foundation*; UNRELATED: Grants/Grants Pending: Other NIH grant support* ( ${ }^{*}$ money paid to institution).

The authors have no potential conflicts of interest with respect to the research, authorship, and/or publication of this article.

\section{REFERENCES}

1. Carey WB, Crocker AC, Coleman WL, et al. Developmental-Behavioral Pediatrics. $4^{\text {th }}$ ed. Philadelphia: Elsevier; 2009:40-49

AJNR Am J Neuroradiol 35:593-98 Mar 2014 www.ajnr.org 
2. Huttenlocher PR, Dabholkar AS. Regional differences in synaptogenesis in human cerebral cortex. J Comp Neurol 1997;387:167-68

3. Shankle WR, Rafii MS, Landing BH, et al. Approximate doubling of numbers of neurons in postnatal human cerebral cortex and in 35 specific cytoarchitectural areas from birth to $\mathbf{7 2}$ months. Pediatr Dev Pathol 1999;2:244-59

4. Barkovich AJ. Brain development: normal and abnormal. In: Atlas SW, ed. Magnetic Resonance Imaging of the Brain and Spine. New York: Raven Press; 1991:129-75

5. Chugani HT. A critical period of brain development: studies of cerebral glucose utilization with PET. Prev Med 1998;27:184-88

6. Brix G, Lechel U, Glatting G, et al. Radiation exposure of patients undergoing whole-body dual-modality 18F-FDG PET/CT examinations. J Nucl Med 2005;46:608-13

7. Detre JA, Wang J, Wang Z, et al. Arterial spin-labeled perfusion MRI in basic and clinical neuroscience. Curr Opin Neurol 2009;22: $348-55$

8. Biagi L, Abbruzzese A, Bianchi MC, et al. Age dependence of cerebral perfusion assessed by magnetic resonance continuous arterial spin labeling. J Magn Reson Imaging 2007;25:696-702

9. Strouse JJ, Cox CS, Melhem ER, et al. Inverse correlation between cerebral blood flow measured by continuous arterial spin-labeling (CASL) MRI and neurocognitive function in children with sickle cell anemia (SCA). Blood 2006;108:379-81

10. Wang J, Licht DJ, Jahng GH, et al. Pediatric perfusion imaging using pulsed arterial spin labeling. J Magn Reson Imaging 2003;18:404-13

11. Fischl B, Salat DH, Busa E, et al. Whole brain segmentation: automated labeling of neuroanatomical structures in the human brain. Neuron 2002;33:341-55

12. Altaye M, Holland SK, Wilke M, et al. Infant brain probability templates for MRI segmentation and normalization. Neuroimage 2008;43:721-30

13. Limperopoulos C, Chilingaryan G, Guizard N, et al. Cerebellar injury in the premature infant is associated with impaired growth of specific cerebral regions. Pediatr Res 2010;68:145-50

14. Blakemore SJ. Imaging brain development: the adolescent brain. Neuroimage 2012;61:397-406

15. Gilmore JH, Shi F, Woolson SL, et al. Longitudinal development of cortical and subcortical gray matter from birth to 2 years. Cereb Cortex 2012;22:2478-85

16. Isaacson J, Provenzale J. Diffusion tensor imaging for evaluation of the childhood brain and pediatric white matter disorders. NeuroImaging Clin North Am 2011;21:179-89

17. Chugani HT, Phelps ME, Mazziotta JC. Positron emission tomography study of human brain functional development. Ann Neurol 1987;22:487-97

18. Smyser CD, Inder TE, Shimony JS, et al. Longitudinal analysis of neural network development in preterm infants. Cereb Cortex 2010;20:2852-62

19. Raichle ME. Behind the scenes of functional brain imaging: a historical and physiological perspective. Proc Natl Acad Sci U S A 1998;95:765-72

20. Detre JA, Rao H, Wang DJ, et al. Applications of arterial spin labeled MRI in the brain. J Magn Reson Imaging 2012;35:1026-37

21. Noguchi T, Yoshiura T, Hiwatashi A, et al. Perfusion imaging of brain tumors using arterial spin-labeling: correlation with histopathologic vascular density. AJNR Am J Neuroradiol 2008;29:688-93

22. Miranda MJ, Olofsson K, Sidaros K. Noninvasive measurements of regional cerebral perfusion in preterm and term neonates by magnetic resonance arterial spin labeling. Pediatr Res 2006;60:359-63

23. Taki Y, Kawashima R. Brain development in childhood. Open Neuroimag J 2012;6:103-10

24. Gogtay N, Giedd JN, Lusk L, et al. Dynamic mapping of human cortical development during childhood through early adulthood. Proc Natl Acad Sci U S A 2004;1:8174-79

25. Galletti C, Kutz DF, Gamberini M, et al. Role of the medial parietooccipital cortex in the control of reaching and grasping movements. Exp Brain Res 2003;153:158-70

26. Piaget JP. The Origins of Intelligence in Children. New York: International Universities Press; 1952:153-236

27. Miller EK, Cohen JD. An integrative theory of prefrontal cortex function. Annu Rev Neurosci 2001;24:167-202

28. Braun $\mathrm{K}$. The prefrontal-limbic system: development, neuroanatomy, function, and implications for socioemotional development. Clin Perinatol 2011;38:685-702

29. Francis JR, Song W. Neuroplasticity of the sensorimotor cortex during learning. Neural Plast 2011;2011:310737 